\title{
Evidenzbasierte Verbandsarbeit: der erweiterte Ethikkodex des Vereins für Socialpolitik
}

\author{
Der neue Ethikkodex des Vereins für Socialpolitik nimmt Stellung gegen die Diskriminierung \\ von Frauen und Minderheiten, sexuelle und anderweitige Belästigung im Arbeitsumfeld \\ sowie das Schaffen eines feindseligen Umfelds. Neuere Untersuchungen zeigen, dass eine \\ Gleichberechtigung von Frauen und Minderheiten in der Volkswirtschaftslehre noch nicht \\ vorliegt. Der erweiterte Ethikkodex ist ein Schritt auf dem Weg zu mehr Chancengleichheit.
}

Was lange währt, wird endlich gut. Während die Vereinigungen der amerikanischen und der europäischen Ökonom:innen schon länger einen Ethikkodex haben, in dem der respektvolle Umgang miteinander thematisiert und spezifiziert wird, fehlte dieser Teil bislang im Ethikkodex des größten deutschsprachigen Fachverbands, dem Verein für Socialpolitik (VfS). Im vergangenen Jahr 2021 hat eine Arbeitsgruppe des Vereins daher einen Vorschlag für die Erweiterung des bestehenden Ethikkodexes erarbeitet. Dieser wurde nun mit gewissen Anpassungen vom Erweiterten Vorstand und den Mitgliedern verabschiedet. Im neuen, erweiterten Ethikkodex werden die Diskriminierung von Frauen und Minderheiten, sexuelle und anderweitige Belästigung im Arbeitsumfeld sowie das Schaffen eines feindseligen Umfelds ausgeschlossen. Dieser Artikel beschreibt die Evidenz, die begründet, dass die Vereinsmitglieder auch in solchen scheinbar selbstverständlichen Punkten eine klare Position einnehmen. Die entsprechende Fachliteratur hat sich zuletzt mit recht eindeutigen Ergebnissen weiterentwickelt.

Schon bei oberflächlicher Betrachtung fällt der hohe Anteil an männlichen Professoren in der Volkswirtschaftslehre auf. Laut Friebel et al. (2021) sind nur $15 \%$ aller VWL-Lehrstühle in Deutschland von Frauen besetzt. Obwohl in den vergangenen Jahren Anstrengungen unternommen wurden, den Anteil an Professorinnen zu erhöhen, scheint sich der Status quo nur sehr langsam zu ändern. In einer aktuellen Studie untersucht Janys (im Erscheinen) die Verteilung der Zahl von Professorinnen

(C) Der/die Autor:in 2022. Open Access: Dieser Artikel wird unter der Creative Commons Namensnennung 4.0 International Lizenz veröffentlicht (creativecommons.org/licenses/by/4.0/deed.de).

Open Access wird durch die ZBW - Leibniz-Informationszentrum Wirtschaft gefördert. an deutschen Universitäten (unter anderem an volkswirtschaftlichen Instituten). Sie zeigt auf, dass es eine „implizite Quote" gibt, die bei ein bis zwei Professorinnen pro Institut liegt. Das bedeutet, dass es durchaus besondere Anstrengungen gibt, Frauen einzustellen, aber dass diese nach dem Erreichen einer geringen Zahl wieder aufgegeben werden. Der Minderheitenstatus von Frauen hat darüber hinaus eine selbstverstärkende Wirkung: Die neuere Literatur belegt, dass bereits Studentinnen ihre VWL-Kurse erfolgreicher absolvieren, wenn sie mit anderen Studentinnen zusammenarbeiten können (Shan et al., 2021), dass Professorinnen oft gebeten werden, Zeit

Prof. Dr. Alexander Kriwoluzky leitet die Abteilung Makroökonomie am DIW Berlin und ist Professor für Makroökonomie an der Freien Universität Berlin.

Prof. Dr. Aderonke Osikominu ist Professorin für Ökonometrie und empirische Wirtschaftsforschung an der Universität Hohenheim.

Prof. Dr. Doris Weichselbaumer ist Professorin und Leiterin des Instituts für Frauen- und Geschlechterforschung der University Linz (JKU) in Österreich.

Prof. Georg Weizsäcker, Ph. D., ist Professor für Volkswirtschaftslehre an der Humboldt-Universität zu Berlin. 
in Auswahlkommissionen zu verbringen, ohne dass dies die Frauenquote bei der jeweiligen Auswahl erhöht (Bagues et al., 2017), und dass Frauen zu Anfragen dieser Art schlechter nein sagen können als Männer (Babock et al., 2017). Auch der Anteil von ethnischen Minderheiten auf Professor:innenniveau ist in der deutschsprachigen Volkswirtschaftslehre sehr gering, auf diese Gruppen könnten daher ähnliche Effekte wirken.

Ganz allgemein ist die Gleichstellung für die Wissenschaft natürlich kein Selbstzweck. Da gerade Wissenschaft von unterschiedlichen Sichtweisen und Hintergründen profitieren kann (Akerlof, 2020), kann es nicht optimal sein, wenn bestimmte Gruppen nur sehr selten in Leitungspositionen aufrücken können. Dementsprechend gilt es zu untersuchen, ob der Grund für den geringen Anteil von Frauen und Minderheiten in den Leitungspositionen eine systematische Benachteiligung ist. Der folgende Überblick liefert Belege dafür, unter Bezugnahme auf neuere Studien zu Geschlechterunterschieden in der Volkswirtschaftslehre.

In den USA ist es in der frühen Karrierephase vor allem wichtig, dass die zunächst befristete Assistenzprofessur in eine dauerhafte Stelle umgewandelt wird. Bereits in einer früheren Studie haben Ginther und Kahn (2004) festgestellt, dass die Chancen, entfristet zu werden, für Frauen in der Volkswirtschaftslehre geringer sind als in anderen Disziplinen. Die Studie diskutiert weiterhin, dass diese ungleiche Behandlung auf keine offensichtlichen oder messbaren Gründe zurückzuführen ist. In einer aktuellen Publikation haben Ginther und Kahn (2021) die Untersuchung erneut durchgeführt und die Ergebnisse weitgehend bestätigt. Ähnlich wie Friebel et al. (2021) es nahelegen, trifft es noch immer zu, dass es für Ökonominnen schwerer ist, entfristet zu werden, als für ihre Kolleginnen in anderen Disziplinen. Allerdings gilt dies vor allem für weniger forschungsintensive Institutionen. Bei den Institutionen mit einem starken Schwerpunkt auf Forschung haben gleichproduktive Frauen auch in der Ökonomik ähnliche Entfristungschancen wie Männer.

In derselben Studie zeigen die Autorinnen auch den Effekt von Publikationen, Zitationen und Drittmitteln auf. Insgesamt können nur ca. 3,5 Prozentpunkte vom Gender Gap in Höhe von 18,5\% in der Entfristungswahrscheinlichkeit auf diese Komponenten zurückgeführt werden. Studien von Sarsons (2017) and Sarsons et al. (2021) untersuchen den Effekt von Publikationen genauer und zeigen auf, dass die Leistungen von Frauen und Männern in gemeinsamen Projekten unterschiedlich wahrgenommen werden. Die Ko-Autorenschaft von Frauen wird dabei geringer bewertet, und eine gemeinsame Publikation zählt bei der Entscheidung über die Entfristung für Frauen weniger als bei einem Mann. Es ist aber nicht nur die Wahrnehmung der Leistung in gemeinsamen Projekten, die es Frauen erschwert, eine dauerhafte Professur zu erhalten. Hengel (2019) zeigt, dass der Publikationsprozess für Frauen erheblich länger dauert als für Männer - geschlagene drei bis sechs Monate länger pro Artikel - in vielen Fällen offenbar deshalb, weil von Frauen geschriebene Papiere einen besseren Sprachstil aufweisen müssen.

Bei den Entscheidungen über Berufungen und Entfristungen spielen zudem nicht nur Publikationen eine wichtige Rolle, sondern auch Lehrevaluationen. Mengel et al. (2019) zeigen, dass das Geschlecht der Lehrenden eine Rolle bei der Evaluation durch die Studierenden spielt. Dozentinnen werden systematisch schlechter bewertet als Dozenten. Hierbei sind die Bewertungen von männlichen Studenten ausschlaggebend, die Dozentinnen schlechter bewerten. Der Effekt ist dabei besonders dann stark ausgeprägt, wenn der Kurs einen hohen Anteil an mathematischen Inhalten aufweist und/oder wenn die Dozentin noch jung ist.

Die schlechten Bewertungen der männlichen Studierenden für junge Dozentinnen spiegeln dabei eine auch allgemein wahrgenommene Atmosphäre wider. Wu (2020) dokumentiert, dass es auf dem Online-Diskussionsforum Economics Job Market Rumors häufig zu sexistischen Äußerungen in Bezug auf Wissenschaftlerinnen kommt. Die dahinterliegende Einstellung mag auch einer der Gründe sein, dass weibliche Vortragende bei Seminarvorträgen öfter unterbrochen werden als Männer. Zwar kann häufiges Nachfragen auch Interesse an einem Thema dokumentieren, allerdings zeigen Dupas et al. (2021), dass die an Frauen gerichteten Fragen eher einen feindseligen bzw. bevormundenden Charakter aufweisen. Weitere Evidenz für die vielfältigen Schwierigkeiten, die sich für Frauen und Minderheiten aus ihrem Arbeitsumfeld ergeben, wurde in einer Umfrage, die von der American Economic Association in Auftrag gegeben wurde, deutlich dokumentiert (Allgood et al., 2019).

Der neue Ethikkodex des Vereins für Socialpolitik ist ein Schritt, um die genannten Missstände aufzuheben. Er kann natürlich nur ein Element in einer Reihe von Maßnahmen sein - Beiträge von Arbeitgebenden und Hochschulpolitik sind ebenfalls gefragt. Zur Verbesserung der Situation von Frauen und Minderheiten können viele aktuell diskutierte Änderungen beitragen, darunter der Ausbau systematischer Nachwuchskonzepte mit glaubhaften Perspektiven (z. B. Tenure-Verfahren), das Abmindern des Lehrstuhlprinzips mit seinen besonderen Abhängigkeitsverhältnissen, sowie weitere Förderungen für Frauen und Minderheiten. 


\section{Literatur}

Akerlof, G. A. (2020), „Sins of Omission and the Practice of Economics, Journal of Economic Literature, 58(2), 405-418.

Allgood, S., L. Badgett, A. Bayer, M. Bertrand, S. E. Black, N. Bloom und L. D. Cook (2019), AEA Professional Climate Survey: Final Report, https://www.aeaweb.org/resources/member-docs/final-climate-survey-results-sept-2019 (2. Februar 2022).

Babcock, L., M. P. Recalde, L. Vesterlund und L. Weingart (2017), Gender Differences in Accepting and Receiving Requests for Tasks with Low Promotability, American Economic Review, 107(3), 714-747.

Bagues, M., M. Sylos-Labini und N. Zinovyeva (2017), Does the Gender Composition of Scientific Committees Matter?, American Economic Review, 107(4), 1207-1238.

Dupas, P., A. Sasser Modestino, M. Niederle, J. Wolfers, und The Seminar Dynamics Collective (2021), Gender and the Dynamics of Economics Seminars, NBER Working Papers, 28494.

Friebel, G., N. Fuchs-Schündeln und A. Weinberger (2021), Statusbericht zum Frauenanteil in der Volkswirtschaftslehre an deutschen Universitäten, Perspektiven der Wirtschaftspolitik, 22(2), 142-155.

Ginther, D. K. und S. Kahn (2004), Women in Economics: Moving Up or Falling Off the Academic Career Ladder?, Journal of Economic Perspectives, 18(3), 193-214.
Ginther, D. K. und S. Kahn (2021), Women in Academic Economics: Have We Made Progress?, AEA Papers and Proceedings, (111), 138-142.

Hengel, E. (2019), Publishing while Female: Are Women Held to Higher Standards? Evidence from Peer Review, https://www.erinhengel. com/research/publishing_female.pdf (2. Februar 2022).

Janys, L. (im Erscheinen), Evidence for a Two-Women Quota in University Departments across Disciplines, Review of Economics and Statistics.

Mengel, F., J. Sauermann und U. Zölitz (2019), Gender Bias in Teaching Evaluations, Journal of the European Economic Association, 17(2), 535-566.

Sarsons, H. (2017), Recognition for Group Work: Gender Differences in Academia, AEA Papers and Proceedings, (107), 141-145.

Sarsons, H., K. Gërxhani, E. Reuben und A. Schram (2021), Gender Differences in Recognition for Group Work, Journal of Political Economy, 129(1), 101-147.

Shan, A.-R., C. Minaudier, B. Alvarez Pereira und S. Chaudry (2021), Gender and Choice over Co-workers: Experimental Evidence, https://ssrn.com/abstract=3958386 (2. Februar 2022).

Wu, A. (2020), Gender Bias in Rumors Among Professionals: An Identity-based Interpretation, Review of Economics and Statistics, 102(5), 867-880.

Title: Evidence-based association work: The extended code of ethics of the "Verein für Socialpolitik"

Abstract: The new Code of Ethics of the Verein für Socialpolitik takes a stand against discrimination against women and minorities, sexual and other harassment in the workplace and the creation of a hostile environment. Recent research shows that equality for women and minorities in economics has not yet been achieved. The expanded Code of Ethics is a step towards greater equal opportunity. 